\title{
A Theorical Model: How Venture Capital and Top Management Team Heterogeneity Influence the Firm Innovation
}

\author{
Chen Hong-an ${ }^{1, a}$, Zhang Xia, b, ${ }^{2}$
}
${ }^{1}$ East China University of Science and Technology Business School, Xuhui, Shanghai, China
2East China University of Science and Technology Business School, Xuhui, Shanghai, China achen1989@ecust.edu.cn, bwuqiongbanre@163.com,

\section{Keywords: Venture Capital; Top Management Team Heterogeneity; Firm Innovation}

\begin{abstract}
Innovation is a hot topic of the times, and innovation drive leads to the development of the first power. Enterprises are facing increasingly fierce competition, the importance of enterprise innovation is once again prominent and imminent; at the same time, with the development and perfection of China's capital market, venture capital plays an increasingly important role in many aspects of enterprise development. An important strategy of implementing innovation-driven development strategy is to use capital market to promote and support innovation. Executives of undercapitalized entrepreneurial enterprises will seek venture capital to maximize the use of capital. We can not help but wonder whether venture capital will have an unusual impact on the innovation of the invested enterprises. Therefore, this paper proposes an exploratory model of the role of venture capital (VC) in enterprise to the innovation based on the heterogeneity of top management team.

\section{一个理论模型：风险资本对企业创新的影响探究一高管团队异质性的 调节}

\author{
陈洪安 $1, \mathrm{a}$, 张霞 $2, \mathrm{~b},{ }^{*}$ \\ 1华东理工大学商学院, 徐汇, 上海, 中国 \\ 2华东理工大学商学院, 徐汇, 上海, 中国
}

achen1989@ecust.edu.cn, bwuqiongbanre@163.com,
\end{abstract}

关键词: 风险资本；高管团队异质性；企业创新

摘要: 创新是时代的热门话题, 创新驱动引领发展第一动力.企业面临的竞争愈发激烈, 企业 创新的重要性又一次被突出且迫在眉睫; 同时, 随着我国资本市场的发展与完善, 风险资本 愈来愈多的作用于企业发展的诸多方面。实施创新驱动发展战略的一个重要策略是利用资本 市场对创新的促进与支持作用，资金不雄厚的创业型企业高管会寻求风险资本，最大化利用 资本, 而我们不禁想问, 风险资本是否会对被投资企业创新有着不一般的影响呢? 因此, 以 高管团队异质性为调节, 本文提出风险资本 (Venture Capital, VC) 对企业创新的作用探究模 型。

\section{1 引言}

21 世纪是一个创新推动的世纪, 创新是推动一个国家经济发展的源泉力量。从 07 年开 始, 本土风险资本逐渐兴起, 根据 CVSource 投中数据终端统计, 2016 年共披露出 1351 支基 
金募集完成，披露的募集完成的规模为 1746.95 亿美元。 ${ }^{[1]} \mathrm{VC}$ 的庞大数额带来的影响已经不 容忽视, 对企业创新的影响也引起了广泛关注; 同时, 在企业管理中, 高管团队都是一个不 容忽视的存在。在高管的众多决策中，必然涉及到风险资本的引进，比如何时引进 VC, 以何 种方式引进风险资本, 是否限制风险资本对企业的管理参与, 等等。鉴于此, 本文的研究目 的就是在考虑到环境动态性的基础上, 讨论风险资本对企业创新的影响, 以及不同的高管团 队如何影响风险资本对企业创新的作用。

\section{2 文献综述}

\section{1 风险资本}

美国风险资本协会将 VC 定义为由职业金融家投入新兴且发展迅速、潜力巨大的企业的 一种权益资本; 风险资本方面专家的定义更强调风险性与收益性，风险资本是投资者寻找具 有高利润、发展潜力大、同时风险也较高的投资机会。 ${ }^{[2]}$ 高建等 (1999) 总结了国际风险资本 的七大特点, 其中提及了风险与收益的全对称, 即承担的风险由收益进行补偿, 风险承担者 即是收益获得者，还包括在风险投资的不同阶段所要求的收益不同；然而，风险投资只在特 定条件下发生, 良好的 VC 是资本、市场、管理、时间、风险投资专业人才、企业家的多元组 合，且 $\mathrm{VC}$ 的最终目的是获得利益并全身而退。[3]

已有相关研究发现，VC 会让被投资企业的人力资源政策、股权激励实施以及雇佣销售和 营销等方面变得更加专业化。 ${ }^{[4]} \mathrm{MarcoDaRin}$ 等（2017）发现 VC 会影响被投资企业的创新战 略, 从而对企业创新造成不同的影响。如果 VC 的合伙人有商业经验, 那么 VC 会更积极地 参与被投企业的经营管理，表现在更加积极地参与雇佣企业高管、帮助企业筹资等方面，同 时跟被投企业的联系也更紧密。[5而更早的 Chemmanur 等 (2011) 发现, VC 的参与增加了被 投企业的销售额，提高了被投企业的生产效率。[6]

有学者认为 VC 能够促进企业创新相关：Kortum 等（2000）发现某一行业 VC 投入的增 长会提高该行业的专利比率。 ${ }^{[7]}$ Arsalan Safari（2017）研究全世界 99 个国家和 83 个行业的历 史数据与专利，探讨风险投资对不同知识产权的影响，在考虑到文化、规制、商业环境与经 济市场状况下，发现风险资本投资对企业创新的正向显著影响。[8]苟燕楠等(2014)研究发现， $\mathrm{VC}$ 对中国企业的研发投入和专利数量有显著的积极影响。[9]李爽（2017）对有 VC 参与的创 业板公司进行研究发现, VC 对技术创新活动确实存在正向激励的作用, 且这个作用会因为 $\mathrm{VC}$ 进入的时机不同而不同, 进入越早, VC 越能促进企业创新。 ${ }^{[10]}$ 陈思等 (2017) 在进一步 研究中发现, VC 促进企业创新的表现之一是企业专利申请数量的显著增长; VC 背景与投资 期限的长短的不同对企业创新的促进效果并不一致。[11]

也有学者认为 VC 对企业技术创新并无影响, Jeng 等 (2000) 指出, VC 进入被投资企业 的目的并非帮助企业创新, 而是使被投资企业上市, 再从中获取风险利益; ${ }^{[12]}$ 陈见丽 (2011) 则认为 VC 的这种利益导向性是短期行为, 并不能为企业带来创新资源, 也就不能促进企业 的创新。 ${ }^{[13]}$ Henry 等 (2016) 发现 VC 对于企业创新并无明显作用, 而是作用于企业如何实 用专利。[14]

\section{2 高管团队异质性}

高层管理团队 (高管团队) 控制着公司的战略方向并影响公司经营绩效, 是公司活动中最 为重要和最有影响力的群体。高管团队的核心要素包括团队构成、团队结构、团队过程 （Finkelstein 等，2008） [15]。团队构成指团队成员的集体特征, 如年龄、资历、专业、教育以 及心理因素包括价值观、认知基础、人格等, 大部分实证研究集中于团队构成的多样性特征, 也即团队异质性（Hambrick 等，2015; Mathieu 等，2014）。[16,17]

Williams 和 O'Reilly（1998）指出高管团队人口特征的差异, 往往会造成成员个体的认知 差异, 并形成内、外两个不同的群体。[18]高阶梯队理论认为,由于战略决策的复杂性和 CEO 的 
有限理性, 高阶领导基于其各自认知和价值观进行战略决策,给组织带来影响, 因此组织成为他 们个人特征的反映。而高管团队成员, 常常用年龄、性别、种族等外部特征的区分变量作为 标准, 选择自己归属的小群体。这些外部异质性形成的小群体容易造成团队内的交流和合作 困难，凝聚力下降等负面效应。

针对高管团队如何影响企业创新的相关研究如下：肖挺等 (2013) 针对服务行业上市公司 进行了研究, 发现高管团队任期的长短不同对商业模式、企业创新绩效等均有正面影响。[19] 高管团队年龄异质性与任期异质性与企业创新效率之间呈现倒 U 型关系, 教育水平异质性与 职能背景异质性对创新效率始终是正向作用，等等。[20]

高管团队成员的异质性是其认知的不同，价值观的差异会使得团队关注不同的信息，整个 团队的视野更加开阔, 因此能更好的识别机会与风险, 更好的进行管理。 ${ }^{[21]}$ 面对环境的动态 变化, 高管团队需要实时调整行为, 进行决策的补充与修正, 不同的特质的高管并不会做出 相同的决策, 而高管团队异质性一方面因为在知识、技能与注意力范围等方面更强而促进决

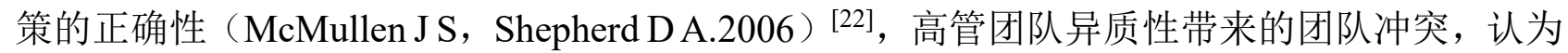
高管团队异质性对企业绩效或创新绩效具有负面影响。虽然认知冲突会带来集思广益的好处, 但是当冲突升级为情感冲突时, 会使整个团队浪费大量的时间和精力用于协调和沟通, 从而 对企业绩效和创新带来负面影响。而在有关 VC 引进决策上, 董静（2016）发现, 高管团队 人力资本特征的不同会导致企业做出不同的风险资本引进策略。

\section{3 研究推理}

\section{1 风险资本与企业创新}

风险资本的最终目的是通过风险投资获得利益甚至是超额的风险收益，在当下创新带动 企业发展、创造利润的环境下，风险投资通过“笁选效应”和“辅导效应”为技术创新带来的积 极影响更加明显 (苟燕楠, 2013)。[9]其中“篮选效应”是指风险投资在选择投资对象的过程中, 能够利用丰富的经验和专业知识克服信息不对称造成的逆向选择问题, 成功筛选出有潜力的 企业，并督促其通过技术创新提高生产效率，最终实现上市，实现 $\mathrm{VC}$ 的目的——收益。“辅 导效应” 是指风险投资一旦进入投资对象后, 便与该企业形成利益共同体, 这将促使风险投资 机构积极为企业发展出谋划策, 为企业的技术创新活动提供信息、市场、融资、知识、人力 资源以及公司治理等方面的辅导和建议，有助于弥补企业在初创期所存在的创新经验不足、 管理落后等问题（Stam W, Elfring T, 2008)。 ${ }^{[23]}$ 因此, 假设 $\mathrm{H}_{1}$ ：VC 会对企业创新效率有正 向作用。具体表现为, 假设 $\mathrm{H}_{1 \mathrm{a}}$ ：VC 的进入会促进被投资企业的创新。假设 $\mathrm{H}_{1 \mathrm{~b}}$ ：VC 持股期 限越长，越能促进被投资企业的创新。

\section{2 高管团队异质性的调节作用}

高管团队年龄异质性。年龄作为个体特征的重要变量, 能够反映阅历、经验积累和心智成 熟程度, 对行为方式、决策偏好、战略选择产生影响, 影响企业创新。随着年龄的增长, 经 验和阅历逐渐丰富, 高管个人对市场规律以及经营经验有着更加全面的掌握, 因此也更有自 信进行创新, 在操作的过程中有着更高的成功率, 可能带来更好的创新。而年龄过大的高管, 随着精力的消耗, 开始出现知识结构老化、学习能力下降、行为决策趋于保守, 受到思维能 力、变通能力、体力脑力退化的限制, 认知结构难以随着社会的发展与时俱进, 面对市场日 新月异的变化也无法快速应对, 因而倾向于固化知识结构、避免风险决策, 从而对企业创新 趋于保守, 其对 VC 促进企业创新的调节作用减弱甚至会出现负效应。因此, 假设 $\mathrm{H}_{2 \mathrm{a}}$ ：高管 团队年龄异质性对于 VC 对企业创新的促进作用呈现出倒 U 型调节。

高管团队职业背景。不同的职业经历带给了高管团队成员不同的认知, 职业背景多元化的 高管团队对外部环境变化有更加敏锐的洞察力, 这样的高管团队对自主创新有更高的认知水 平和更多关注。管理者的职业经验会影响组织的战略选择，同时，高管的职业背景在一定程 
度上反应其偏好、信息处理，也影响到战略选择与决策。这些不同的经验与认知使得高管团 队成员思考角度不同, 解决问题的方案更加趋向多样化, 另一方面, 彼此不同就能相互学习 与交流，带来高管团队内部的成长而最终作用于对于 $\mathrm{VC}$ 的选择、相关创新决策、以及利用 $\mathrm{VC}$ 对企业创新的促进作用上。职业背景多样化的高管团队能更大化的利用 VC 的资本作用, 强化 VC 对企业创新的促进。因此, 假设 $\mathrm{H}_{2 \mathrm{~b}}$ ：高管团队职业背景异质性正向调节 VC 对企业 创新的促进作用。

高管团队任期。任期是指高管在企业内部担任影响企业运营管理等岗位的在岗时间。在任 职初期，高管对企业缺乏全面了解，管理与决策相对保守，高管会花费更多的时间和精力来 熟悉企业的各方面, 而从进入创新活动到拥有创新产出是需要时间的。因此, 高管任期很短 时, 不大可能会表现强烈创新的信号。而随着时间推移, 高管对企业的适应性提高, 对企业 内外部环境的了解更多, 能更清楚的了解企业的长处与短处, 可以针对性的推进创新战略从 而促进企业成长。因此, 本研究认为随着高管团队成员的任期增加, VC 之于企业创新的促进 作用会加强。因此，本文假设 $\mathrm{H}_{2 \mathrm{c}}$ ：高管团队任期正向调节 VC 对企业创新的促进作用。

\section{4 模型提出}

基于前文论述， $\mathrm{VC}$ 在选择被投资企业时，被投资企业的高管团队本身的特征是 $\mathrm{VC}$ 的一 大考虑因素, 而高管团队对决策过程的参与也在一定程度上影响 VC 对被投资企业创新的影 响。由由此, 本文如下图所示的理论模型。

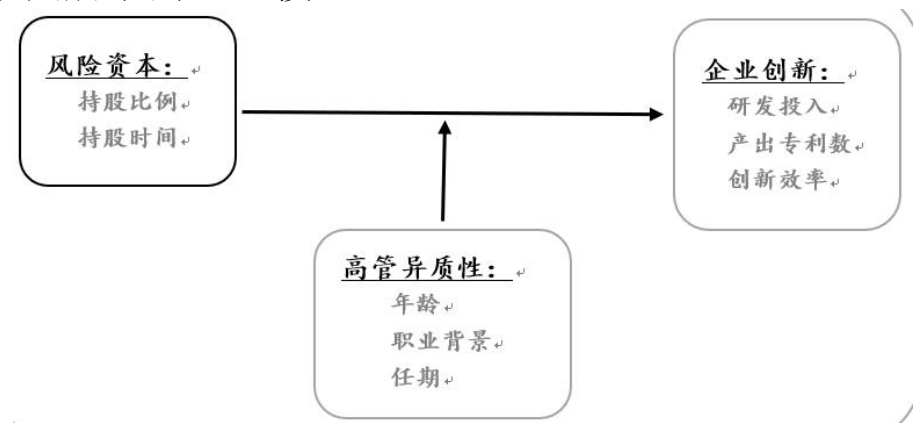

图 1 理论模型

\section{References}

[1] Investing statistics: 2016 domestic VC investment amounted to 44.9 billion US dollars; 2017. http://finance.sina.com.cn/stock/t/2017-01-10/doc-ifxzkfvn1339298.shtml.

[2] Feng Xiaoli, Shi Ping. Summary of Foreign Venture Capital Market Theory Research[J]. Economic Issues, 2005(05):17-19.

[3] Gao Jian, Chen Song. Seven Characteristics of International Venture Capital [J]. China Soft Science, 1999(03):36-39.

[4] Hellmann, T. and M. Puri. Venture Capital and the Professionalization of Start-Up Firms: Empirical Evidence[J].The Journal of Finance.2002, Vol.57, pp.169-197.

[5] Marco Da Rin, María Fabiana Penas. Venture capital and innovation strategies[J]. Industrial and Corporate Change.2017, Vol.26(No.5): 781-800.

[6] Chemmanur, Thomas J.1;Krishnan, Karthik2;Nandy, Debarshi K.3.How Does Venture Capital Financing Improve Efficiency in Private Firms? A Look Beneath the Surface[J]. Review of Financial Studies.2011, Vol.24(No.12): 4037-4090.

[7] Fitza M, Matusik S F, Mosakowski E. Do VCs matter? The importance of owners on 
performance variance in start - up firms[J]. Strategic Management Journal, 2009 (4):38 - 40.

[8] Arsalan Safari. Worldwide venture capital, intellectual property rights, and innovation. Industrial and Corporate Change[J]. 2017, Vol.26(No.3): 485-515.

[9] Yan Yannan, Dong Jing. Research on the impact of venture capital background on enterprise technology innovation [J]. Scientific research management, 2014 (2):35 -42.

[10]Li Shuang. Does venture capital increase the enthusiasm of Chinese enterprises for technological innovation? —_Based on the "double substitution effect" under the background of capital price distortion[J].Journal of Xi'an Jiaotong University(Social Science Edition). 2017, (2): 17-23, 75 .

[11]Chen Si, He Wenlong, Zhang Ran. Venture Capital and Enterprise Innovation: Impact and Potential Mechanism [J]. Management World.2017, (1): 158-169.

[12]Jene L A, Wells P C. The determinants of venture capital funding: Evidence across countries[J]. Journal of Corporate Finance, 2000 (3):241 -289.

[13]Chen Jianli. Can venture capital promote technological innovation in high-tech enterprises? -Based on empirical evidence of China's GEM listed companies [J]. Economic management,2011(2):71 - 77.

[14]Henry Lahrab, Andrea Minac. Venture capital investments and the technological performance of portfolio firms[J].Research Policy.2016,Vol.45(No.1): 303-318.

[15] Sydney Finkelstein, Donald C. Hambrick, Albert A. Cannella， Jr. Strategic leadership: theory and research on executives, top management teams, and boards[J]. Oxford University Press, USA, 2009

[16]Hambrick Donald C, Humphrey, Stephen E, Gupta Abhinav. Structural interdependence within top management teams: A key moderator of upper echelons predictions[J].Strategic Management Journal.2015, Vol.36(No.3): 449-461.

[17]John E. Mathieu;Scott I. Tannenbaum; Jamie S. Donsbach; George M. Alliger. A Review and Integration of Team Composition Models: Moving Toward a Dynamic and Temporal Framework[J]. Journal of Management.2014, Vol.40(No.1): 130-160.

[18]K Y Williams, Cao Iii. Demography and Diversity in Organizations: A Review of 40 Years of Research[J]. Research in Organizational Behavior.1998, 20 (3) :77-140.1998

[19] Tanikawa T, Kim S, Jung Y. Top management team diversity and firm performance: exploring a function of age[J]. Team Performance Management, 2017, 23(3/4).

[20]Han Qingwei, Yang Chen, Gu Zhipeng. The Threshold Effect of Executive Team Heterogeneity on Enterprise Innovation Efficiency_An Empirical Study Based on Strategic Emerging Industries Listed Companies[J]. China Economic Issues.2017, (2): 42-53.

[21]Ma Fuping, Guo Xiaochuan. Research on the Relationship between Executive Team Heterogeneity and Technological Innovation Performance: Taking Executive Team Behavior Integration as a Regulating Variable [J]. Science and Science and Technology Management.2010, (12): $186-191$.

[22] McMullen J S, Shepherd D A. Entrepreneurial action and the role of uncertainty in the theory of the entrepreneur[J]. The Academy of Management Review, 2006.

[23] Stam W, Elfring T. Entrepreneurial orientation and new venture performance: The moderating 
role of intra-and extra-industry social capital[J].Academy of Management Journal.2008,51:97 -111 . 